\title{
LA RADIO A LA ESCUELA
}

\author{
Jesús Jiménez Martínez \\ Maestro del CRA Cameros Nuevo
}

\begin{abstract}
RESUMEN. Muchas son las razones por las que, en mi opinión, se debe introducir el uso sistemático de los Medios de Comunicación en las aulas de nuestros centros educativos. Ahora bien, para que surta los efectos oportunos esta actividad ha de ser convenientemente planificada. Por otro lado, es importante que los docentes responsables de su introducción conozcan a la perfección el medio o medios que van a trabajar en el aula. Valores como la tolerancia, el respeto a la opinión del otro o la libertad de expresión tienen su pleno sentido en el desarrollo de esta actividad. Podemos dar una lección magistral de cómo ser tolerantes y respetuosos con los demás, pero realmente aprenderemos a serlo si lo vivimos y lo practicamos a diario.

A decir verdad, muchos docentes se plantean el trabajo con estos medios, pero encuentran dificultades a la hora de ponerlos en práctica. Este artículo pretende ser una reflexión sobre diferentes aspectos a tener en cuenta a la hora de trabajar con ellos en el aula y, por otra parte, intenta dar pistas sobre diferentes actuaciones que podemos llevar a cabo en nuestras aulas.

ABSTRACT. In my opinion, there are many reasons for the introduction of systematic use of the media in the classrooms of our schools. But if we want to have the desired effect, this activity must be well planned.

On the other haud, the teachers who want to carry forward this plau should know everything about mean or means that they're going to handle. In the course of the development of this job, the tolerance, the respct for the other people's opinions and the freedom of speech get a special meaning; we can give a master class about how to be tolerant and respectful but this values won't be learned if we don't practice them every day, if we don't create the right asmosphere.

To tell the truth, many teachers would like to work about and through the media but they find a lot of problems in his way. Thir article tries to be a reflection about all the different aspects of this kind of job. it tries as well to give some trails about different actions we can develops in the course of our lessons.
\end{abstract}

\section{Los medios de comunicación en el aula}

\author{
"El $80 \%$ de la información que los alumnos/as reciben en las aulas no les \\ sirven, el 80 \% de la información que manejan los alumnos/as la reciben de \\ fuera del centro". (Lemaresquier)
}

Sólo esta razón sería suficiente para poner en marcha en nuestras aulas el estudio serio y riguroso de los medios de comunicación en sus diferentes facetas. Sin embar- 
go, existen una cantidad ingente de motivos para hacer llegar a lo centros ese cuarto poder que, en estos momentos más que nunca, conforman ideologías, hábitos, valores e, incluso, me atrevería a decir, transforman conductas.

Nos encontramos inmersos en el mundo de la imagen y de la comunicación. El bombardeo de noticias que la sociedad recibe es continuo, el mundo se acerca gracias a los medios de comunicación. La invasión de éstos y otros nuevos que se nos acercan están provocando importantes cambios a todos los niveles.

Es muy importante la influencia de estos medios en todas las capas de la sociedad, pero especialmente sobre los elementos más fáciles de transformar por encontrarse en proceso de formación, los niños y jóvenes. Todos somos conscientes de la influencia, positiva o negativa, de la televisión en nuestros alumnos/as. No es el momento de profundizar en este aspecto por cuanto sería reiterativo y todos estamos de acuerdo en ello.

Es por tanto necesario que nuestros alumnos/as sean educados en este campo como uno más de su desarrollo integral, que sepa hacer un buen uso de estos medios, que sepa entender, comprender las noticias, seleccionarlas, criticarlas, asimilarlas, que conozca las relaciones de la sociedad, situación, los problemas del mundo actual. Por ello y para ello debemos informarle, formarle, ayudarle y animarle a hacer uso de estos medios de comunicación de una manera correcta y en función de sus intereses.

Partiendo del principio pedagógico que afirma que HACER es mejor que ESTUDIAR, será mejor hacer radio, prensa y televisión que no sólo estudiarlos sistemáticamente. Haciendo se aprende a valorar, a criticar de manera constructiva y a seleccionar aquello que realmente nos sirva para nuestra propia formación.

Los medios de comunicación cuentan lo que pasa en el mundo, pero no pasa sólo lo que ellos cuentan y cómo lo cuentan. Esto implica que los centros deben evitar, formando a su alumnado, que éstos sean manipulados. Todos los medios de comunicación tienen su ideología a pesar de que se consideren o manifiesten su independencia. Esto supone que los centros deben hacérselo comprender a su alumnos/as para que actúen en consecuencia.

Existen multitud de medios de comunicación, todos se pueden y deben estudiar. Sería importante que los centros los integrasen en su curriculum de tal forma que, al finalizar la etapa escolar obligatoria, sus alumnos/as fueran conocedores de todos ellos con un mínimo de profundidad.

No obstante todo lo anterior, existen otras razones mucho más poderosas para hacer uso de los medios de comunicación en el aula.

Una de ellas tiene que ver con la formación integral del alumno, con su formación personal sobre todo. Es un elemento imprescindible para que el alumnado forme su propia opinión y desarrolle el sentido crítico del que tantas veces hablamos en educación y que en pocas ocasiones intentamos trabajarlo por razones que no vienen a cuento. Valores como el respeto a la opinión de los demás, la tolerancia, de la que tanto hablamos, libertad de expresión, tiene su razón de ser en el trabajo con los medios de comunicación en nuestros centros. Todo esto puede explicarse de una manera teórica y, con toda seguridad, daremos una excelente clase magistral, pero eso no es suficiente. Ponerlo en práctica es la manera y forma como nuestros alumno/as lo van a entender, asimilar y asumir.

El trabajo con los medios será, además, un elemento importante para que el alumnado comprenda la realidad que le rodea. Es la fórmula para que se den cuenta de la 
importancia de la realidad actual como configuradora de la sociedad contemporánea en la que ellos tienen su palabra, su opinión y actuación.

Otra razón, asimismo importante, tiene que ver con el aspecto pedagógico. Estamos escuchando continuamente hablar de la importancia del estudio del entorno para nuestro alumnado. Estudio del entorno próximo con el fin de conocerlo, expresarlo y, sobre todo, poder transformarlo. Por otro lado, estos medios son una forma de apertura de los centros y un cauce importante de expresión. Los centros han de estar abiertos al exterior, al barrio, a la localidad. Estos medios son uno de los elementos de relación entre el centro y la sociedad. Es una posibilidad para que la sociedad más cercana al centro, la familia, se implique en la educación de sus individuos. En muchas ocasiones la escuela puede ser un foco importante de cultura en el pueblo, a veces el único. Eso ha de aprovecharlo en beneficio de los alumnos/as del centro.

Introducir los medios de comunicación en los centros supone, además, hacer una escuela diferente. Escuchamos en muchos foros, charlas, debates y cursos de formación, la importancia de hacer uso en nuestras aulas de una metodología activa, participativa, abierta, investigadora, innovadora y motivadora. Sin duda alguna, el uso de estos medios es una de las estrategias. Esto supone, claro está, mucho más esfuerzo, tiempo y trabajo, sobre todo para el profesorado. Pero el nivel de motivación que alcanza nuestro alumnado es bastante elevado. Se trata de hacer girar muchas de las actividades que se proponen en el aula en torno a los medios o al medio que cada uno o centro seleccione. Todo lo que se trabaja en las aulas es susceptible de ser utilizado en los medios de comunicación, lo cual confiere a las actividades un nivel de motivación importante añadido.

Por otro lado y centrándonos en el área de Lenguaje, podemos decir que la mayor parte de los bloques de contenido tienen cabida en el estudio de los medios. Todas las materias salen reforzadas, pero, lógicamente, de manera muy especial el área de Lenguaje. Todos hemos oído multitud de veces que nuestros alumnos y alumnas no saben estudiar, no saben hacer resúmenes, esquemas, en definitiva, que no saben hacer uso de las técnicas de estudio. Con estos medios, los alumnos/as hacen uso de todas esas técnicas: resumir, subrayar, componer, descomponer, leer, dramatizar, crear, comunicar, prestar atención, colaborar, cooperar, perder el miedo a hablar, perder la timidez, mejorar la dicción,...

Por tanto, si comprendemos la importancia de los Medios de Comunicación en nuestra sociedad actual, si somos conscientes de su importancia en la formación integral de nuestros alumnos/as, si entendemos que su uso en las aulas supone un alto nivel de motivación y si consideramos que muchas de las áreas del curriculum sufren una gran avance con ellos, en especial el área de Lenguaje, hemos de ser consecuentes con todo ello y, por lo tanto, hemos de introducir en nuestras aulas el estudio de estos Medios de Comunicación.

A pesar de todo lo anterior, es necesario andar con relativo cuidado puesto que podemos caer en una serie de errores que den al traste con todos los aspectos positivos que acabamos de citar. Uno de ellos puede ser la introducción de la prensa en las aulas como único medio de comunicación. Existen otros muchos que, además, son más influyentes que la prensa escrita. El hecho de que los periódicos sean más fáciles de trabajar en el aula por razones evidentes, no quiere decir que sea el medio a trabajar en exclusiva. Será importante llevar a nuestras aulas también la televisión y el vídeo, la radio, internet, etc. 
Otro error, más grave que el anterior, sería centrar toda la actividad de nuestras aulas en los medios de comunicación. No todo está en ellos. Éstos son y han de ser un excelente complemento a nuestra actividad diaria.

\section{2. ¿Cómo introducir los medios en el aula?}

No podemos introducir el uso y estudio de los Medios de Comunicación en nuestras aulas de cualquier forma y manera. Es necesario que nos paremos a reflexionar largo y tendido sobre determinados aspectos que, en especial, el profesorado debe tener en cuenta.

Uno de los primeros elementos a tener en cuenta tiene mucho que ver con la improvisación. Sin lugar a dudas, uno de los mayores errores de cualquier docente es el hecho de improvisar en el aula. No preparar la clase, no tener organizadas las actuaciones a llevar a cabo en cada momento, nos lleva a una situación muy delicada. Es un engaño a nuestro alumnado. En el caso de los Medios es muy importante que el profesorado esté bien formado e informado sobre ellos. De poco servirá a nuestro alumnado trabajar con los medios, si quien les debe preparar apenas los conoce. Si no estamos preparados, si no sabemos, si desconocemos los Medios, poco podemos transmitir a nuestros alumnos y alumnas sobre estos temas.

Por otro lado, es importante, como en cualquier otra materia, que el trabajo esté convenientemente programado. Esta actuación con los Medios de Comunicación, ha de quedar integrado en la programación del curso escolar. No puede quedar al arbitrio de no se sabe qué. No puede quedar como opción a momentos o tiempos muertos. Ha de ser un estudio serio y riguroso.

Otro elemento a reflexionar tiene que ver con qué Medios de Comunicación podemos introducir en las aulas. En principio, todos ellos son susceptibles de ser trabajados en el aula. Seremos nosotros quienes, en función de una serie de criterios, decidamos cuál o cuáles hemos de trabajar. Sin duda, la prensa es el más socorrido en las aulas, lógicamente por sus características. Es, ante todo, un medio individual y tranquilo. No obstante, a la hora de seleccionar el medio a trabajar, debemos tener en cuenta otros aspectos. Los planteamientos educativos que cada uno de nosotros, como docentes, tenemos son un elemento a tener en cuenta. Estos nos llevarán a participar o no de todo lo que estamos indicando ya que no podemos olvidar que, como he citado al comienzo del artículo, el uso de estos medios supone un cambio radical en el aula a nivel, sobre todo, metodológico. Nuestra propia formación es, sobre todo, un elemento fundamental. Poco podemos transmitir si desconocemos el medio. El interés del profesorado por uno u otro también marcará nuestra selección. Si el alumnado se mueve por interés, no es menos el caso del profesorado. Si no creemos en ello, es bastante difícil que podamos contagiar algo a nuestro alumnado. Junto a estos, existen otros elementos tan importantes como los anteriores. Es el caso de las posibilidades económicas de que dispongamos en el centro. Comprar el periódico a diario, realizar programas de vídeo y hacer el montaje, montar una emisora de radio, todo ello requiere de unos recursos económicos de los que se carecen en la mayoría de los centros. No obstante, es el momento de poner en marcha la imaginación para poder solucionar este problema. Participar en proyectos de Innovación Educativa, pedir subvenciones a Consejerías, Ayuntamientos, APAs, etc. puede ser una de las soluciones. Aspectos organizativos como los tiempos o espacios pueden marcar la selección de uno u 
otro medio. Todos los Medios pueden ser utilizados, incluso, al mismo tiempo. Cada uno aporta sus propias características. Cada uno de ellos tiene elementos suficientes para motivar a unos o a todos nuestros alumnos y alumnas.

Cómo introducir estos Medios, requiere una última reflexión. Es imprescindible decidir qué aspectos hemos de trabajar en nuestras aulas. Tres son las posibilidades educativas: CONOCER, USAR Y ELABORAR. En mi opinión, las tres hemos de incluir en el estudio serio y riguroso que estamos predicando desde el comienzo.

"CONOCER" supone la parte teórica del trabajo con los Medios de Comunicación. Es, sin duda, una parte importante e interesante que ha de verse complementado por las otras dos posibilidades. El profesorado ha de conocerlo previamente. Este estudio puede iniciarse en los últimos cursos de Primaria y ha de profundizarse durante la E.S.O. Se hace necesario conocer cómo es el medio, cómo funciona, cómo transmite el mensaje y cómo influye en el receptor. Para llevar a cabo este estudio, es imprescindible tener en cuenta todo lo que hemos indicado acerca de la improvisación, integración de contenidos y programación.

"USAR" es más motivador para el alumnado en general. Los Medios son un instrumento didáctico seductor para temas generales e interdisciplinares. En muchos momentos y en relación con muchos contenidos podemos echar mano de ellos para complementar la información de los libros de texto. Todas las áreas del curriculum se manifiestan de una u otra manera en los medios. En mi opinión, los diferentes contenidos a trabajar en nuestras aulas tienen su correspondencia en la actualidad, y ésta es un elemento motivador para nuestro alumnado. Estos Medios nos aportan un material variado, actual y sugerente. Ahora bien, hemos de tratarlo correctamente y en el momento oportuno. Las posibilidades son inmensas. Sin embargo, la mejor de todas es el estudio de la actualidad a todos los niveles. En la Primaria tiene cabida el estudio de lo más cercano: lo local, lo comarcal, lo regional. En los cursos superiores nos acercamos a lo más lejano, la actualidad nacional e internacional. En este caso, los Medios de Comunicación serán la motivación para que se interesen por todo lo que nos rodea, por la actualidad, por la sociedad, por ellos mismos. Son un lugar donde acudir en busca de información para la elaboración de temas monográficos, informes, trabajos de fondo...

"ELABORAR" diferentes programas de radio o televisión y editar revistas o periódicos escolares es una de las mejores fórmulas para el aprendizaje sobre los Medios. Es el momento culminante de todo el trabajo con ellos. Es el instante donde aparecen las verdaderas dificultades que hemos de solventar con ayuda de la aportación teórica. Es la situación óptima para tomar conciencia de aspectos claves en el mundo de los Medios de Comunicación: ideología, censura, independencia, financiación, libertad de expresión, críticas, manipulación, intoxicación, publicidad, respeto a la opinión del otro, tolerancia. Todo ello es aprehendido mediante la elaboración de los diferentes medios de comunicación. La experiencia diaria de trabajo en el aula sobre este aspecto particularmente ha sido muy enriquecedora para todos, tanto alumnado con el que he trabajado a lo largo de estos años como para mí mismo. Existen multitud de posibilidades a la hora de trabajar este aspecto. Elaborar diferentes tipos de programas de radio, de los que hablaremos más adelante con mayor profundidad, confeccionar diversos tipos de periódicos escolares o producir cualquier programa de televisión, está al alcance de nosotros y de nuestros alumnos y alumnas que, a buen seguro, estarán encantados y entusiasmados con la experiencia. 


\section{Una emisora de radio escolar}

Sin duda, uno de los medios que más posibilidades educativas nos ofrece es la radio escolar. Es un medio complejo para introducir en nuestros centros, pero es el medio más enriquecedor desde el punto de vista pedagógico.

Mi experiencia personal con la radio escolar se remonta al curso 85/86 en una escuela rural de un pequeño pueblo situado al sureste de la comunidad Autónoma de La Rioja Ilamado Cabretón. Fueron dos años inolvidables por muchas razones, una de ellas nuestra emisora conocida como Radio-Escuela. Posteriormente seguí trabajando con la radio en dos situaciones diferentes. En un primer momento monté una emisora escolar en el desaparecido Centro de Recursos de Torrecilla que nos sirvió para realizar emisiones en algunos pueblos como Villoslada, Torrecilla, Montenegro y Sotés. Era el curso 87/88. Los años siguientes estuve trabajando en Logroño, lo cual me impidió, como es lógico y comprensible, poder realizar emisiones al exterior, aunque seguí trabajando esta misma actividad bajo otros planteamientos. Años después, en el curso 98/99, empiezo una experiencia similar a la de Cabretón en otro centro rural perteneciente al CRA "CAMEROS NUEVO". Se trata de Villoslada de Cameros donde surge la emisora escolar denominada "RADIO VILLOSLADA". Desde el curso 2000/01 hasta la actualidad hemos puesto en marcha la emisora en el Colegio Público de Ortigosa y, lo más importante, con la autorización correspondiente del Ministerio de Ciencia y Tecnología, siendo nuestra emisora la única de España en contar con la citada autorización gracias al apoyo recibido desde la Consejería de Educación y de Administraciones Públicas especialmente.

Poner en marcha una emisora de radio escolar parece que tiene que ser muy complicado. Nada más lejos de la realidad. Todo nace por intuición, por un impulso. Cae en mis manos una emisora de radio de FM y automáticamente, será por deformación profesional, la sitúo en mi centro, en mi aula y para mis alumnos y alumnas. En principio no pienso en nada más. En verdad, se me empieza a caer el mundo cuando el día 8 de enero de 1985, a la vuelta de las vacaciones de Navidad, comenzamos a emitir sin saber hasta dónde podríamos llegar con este invento. Han pasado 15 años. Recuerdo o mejor todos recordamos aquellos primeros días con un cariño muy especial. Fue una experiencia inolvidable que nos unió mucho como grupo. Fue una actividad que marcó a todos los alumnos y alumnas que participaron. Fue un hito en la historia del pueblo.

Durante estos años, he reflexionado con cierta calma y muchas veces sobre esta actividad. He asistido en varias ocasiones a dar charlas sobre el tema, lo cual me ha llevado a plasmar oralmente y por escrito toda una serie de aspectos que, aunque yo no lo hice, deberíamos analizar antes de iniciar una actividad semejante. No obstante, a veces pienso que si hubiera tenido que analizar previamente todo lo que a partir de ahora voy a comentar, acaso nunca habría llevado la radio a la escuela. Creo que lo fundamental para innovar en la escuela es tener ganas de aprender, tanto unos como otros. O quizás no haga falta innovar, pensarán otros.

\subsection{Hacer radio}

Antes de lanzarse a trabajar con este medio en el aula haciendo programas de radio, nos debemos plantear la forma en que lo vamos a hacer. Tenemos varias opciones y todas ellas son igualmente válidas en función de las necesidades y, sobre todo, los objetivos a conseguir. 
Una primera fórmula, que es la más sencilla y la más barata, es utilizando el radiocassette. Éste lo utilizaremos para grabar el programa realizado y posteriormente escucharlo en nuestra aula y con nuestros alumnos y alumnas. El resultado, con toda seguridad, será espectacular y nuestros alumnos, a la larga puesto que es un proceso de trabajo, mejorarán sobre todo en muchas facetas del área de Lenguaje y de otras materias como Sociales o Conocimiento del Medio, Naturales, etc.

Una segunda posibilidad nos la puede brindar la megafonía interior del centro que casi todos los colegios poseen. En la mayoría de los centros y sus correspondientes aulas hay un altavoz que nos permitiría, en un momento dado, realizar emisiones ya sean grabadas o bien en directo. Los resultados obtenidos serían, a la larga y como en el caso anterior, espectaculares también. La mejora, con relación al radio-cassette, estaría en el campo de la técnica y especialmente de la motivación del alumnado ya que toda la actividad va más allá de su propia aula y de sus compañeros de pupitre puesto que todo el centro podría escuchar el programa elaborado.

Por último, nos encontramos con la emisora escolar que emite al exterior en las mismas condiciones y características que una emisora comercial. En este caso la motivación del alumnado es clara y evidente, sus propios padres y su gente le va a poder sintonizar y escuchar. Por ello con casi toda seguridad, los objetivos relacionados con el mundo de la comunicación y del lenguaje están conseguidos de antemano. El problema se plantea con otros temas: la legalidad vigente, las cuestiones económicas, los tiempos de dedicación, los espacios necesarios y el entorno cercano o pueblo, entre otros muchos.

El primero de los problemas con el que nos tenemos que enfrentar es la legalidad vigente que no vamos a discutir. Para tener una concesión de una emisora de Radio de FM hay que esperar los plazos marcados por la ley, solicitar una de ellas al igual que lo hace una emisora comercial y esperar la resolución del Gobierno de turno. Con este planteamiento no merece la pena ni seguir tratando este tema puesto que no existe ninguna posibilidad real de que a un centro escolar le concedan licencia alguna. Existen otras opciones. Se puede solicitar como emisora Cultural, posibilidad que brinda la legislación de La Rioja para Asociaciones sin ánimo de lucro o, poniéndose de acuerdo con el Ayuntamiento de la localidad, solicitarla como emisora municipal. En el caso de Radio Villoslada solicitamos, como primer paso, una ayuda económica al MEC, curso 98/99, como Proyecto de Innovación Educativa. Desde el Ministerio se nos concedió todo el dinero que presupuestamos para ponerla en marcha. Ese primer curso estuvimos trabajando sin ninguna traba. Al finalizar el curso escolar 98/99, una vez asumidas las transferencias de Educación por el Gobierno de La Rioja, recibimos una comunicación de la Consejería de Educación que nos apremiaba a pedir los correspondientes permisos previa presentación de una serie de documentación y proyecto correspondiente. Nuestra intención, como CRA de Cameros, era ampliar el radio de acción de la emisora escolar y que se pudieran beneficiar de esta excelente y pedagógica actividad todo el alumnado de la sierra camerana. Nuestra sorpresa fue mayúscula cuando se nos informó, por parte de la Consejería de Administraciones Públicas de La Rioja, que no podíamos seguir realizando la citada actividad escolar por carecer del consiguiente permiso, pero que se pondrían en contacto con el Ministerio de Ciencia y Tecnología a fin de recabar su autorización. Y así fue. Nuestra sorpresa fue enorme cuando se nos informó, allá por el mes de mayo de 2001, de que teníamos la autorización correspondiente, aunque debíamos cumplir una serie de requisitos: emisión quincenal, con un tiempo máximo de 15 minutos, etc... 
Las cuestiones económicas suponen un problema añadido a la actividad pero que, en mi opinión, no pueden ser una rémora en el desarrollo de la misma. Hemos de ser imaginativos e intentar buscar fondos donde los hay. En la primera época, RADIO ESCUELA DE CABRETÓN, no tuvimos dinero y lo hicimos con la aportación de los medios técnicos que teníamos en nuestras casas. La emisora, el único micrófono, y los dos radio-cassettes eran de unos y otros. Adquirimos sólo y exclusivamente lo necesario e imprescindible: cables y enchufes. En la segunda etapa, RADIO VILLOSLADA, con todo el bagaje de conocimiento que tenía por al experiencia acumulada, realizamos, como ya he citado anteriormente, una solicitud al MEC como proyecto de Innovación Educativa. En este caso realicé un proyecto muy completo de necesidades para montaje de la emisora. Al tiempo se nos informó de la concesión, íntegra, de todo el presupuesto. Con ella hemos estado trabajando durante un curso escolar. Recibimos, además, ayuda del propio CRA.

Para la instalación de la emisora en el C.P. de Ortigosa hemos contado con la ayuda del APA y del ayuntamiento. En el tema económico, las posibilidades son muchas, se trata de buscar.

Dejando de lado los aspectos externos a la actividad educativa y centrándonos en lo meramente pedagógico, existen una serie de reflexiones que, como docentes responsables, tenemos que realizar. Un primer elemento de reflexión tiene que ver con los tiempos a dedicar a esta motivadora actividad. Por un lado tenemos los momentos en que nuestros alumnos y alumnas han de elaborar los programas correspondientes: buscar, seleccionar, redactar, poner música, grabar. Por otra, la emisión misma. Lógicamente, esta actividad forma parte del curriculum de diferentes materias y por tanto nadie puede pensar, o no debería pensar, que resta a los contenidos de determinadas asignaturas. Se trata de analizar qué momentos de la semana y en qué materias podemos llevar a cabo estas actividades. Mi experiencia personal comienza con una serie de horas extraescolares en el colegio de Cabretón. Lo hacíamos todos, alumnado y yo mismo, con muchas ganas e ilusión. Sin embargo, al curso siguiente la reflexión fue muy simple: "si esta actividad es tan motivadora y tan rica pedagógicamente hablando, la vamos a incluir como una más dentro del horario del alumnado". De esta forma, dedicábamos una sesión semanal del área de Lenguaje, concretamente los jueves que era día de emisión, a realizar los diferentes programas. Nadie ni nada se resintió. En el caso de Villoslada repetí el mismo planteamiento y dedicamos una sesión semanal al tema. Como se puede comprender, el alumnado y yo mismo dedicábamos muchas más horas en nuestras respectivas casas a la elaboración de los correspondientes programas, pero esas no las contamos. En estos momentos y en la ESO tenemos una asignatura denominada Procesos de Comunicación en la que realizamos la elaboración de los guiones de los diferentes programas.

En cuanto a los espacios necesarios para la emisión de los programas, podemos hablar de lo ideal y de lo real. Lo ideal es conocido por todos: locutorio y sala de control. Sin embargo, eso se encuentra lejos de las posibilidades, al menos de lo que son los centros rurales donde yo me he movido. Existen centros educativos en otras Comunidades Autónomas que no han tenido ningún tipo de problema a este respecto y que además están reconocidos por las propias instituciones como algo digno de mencionar, que poseen verdaderos estudios de radio. En nuestro caso no pedimos tanto. Nos hemos conformado siempre con las emisiones desde nuestra propia aula y no hemos puesto reparo a que los elementos técnicos estén a nuestro alrededor. Resulta incluso romántico. 
Un aspecto que debemos analizar a conciencia es la relación de la emisora escolar y educativa con el medio donde se va a desenvolver, en nuestro caso los municipios o pueblos. Si importante es esta actividad para el progreso y desarrollo de nuestro alumnado, puede ser muy útil para el pueblo. Se ha hablado y seguimos hablando algunos de la escuela como foco cultural de los pueblos. Se comenta la importancia que un colegio tiene para la vida y la continuidad de un pueblo. Se habla de la formación continua, aquella que no acaba nunca pero que en los pueblos apenas llega. Se afirma de la necesidad de las "Escuelas de Padres". Se dice mucho de la falta de actividades culturales en los pueblos para los jóvenes. Aquí tenemos una posibilidad para ser explotada. Ahora bien, desde los centros educativos hemos de ir con mucho cuidado y recelo. Hemos de reflexionar ampliamente sobre todos los aspectos que he señalado y otros muchos que se pueden plantear. Al final de esa reflexión, saldrá toda una serie de actuaciones y estrategias o, por el contrario, se decidirá que no merece la pena. Lo fundamental es pensar en todo ello y decidir. No podemos ni debemos olvidar, sin embargo, las consecuencias que de la citada decisión se derivarán posteriormente, para bien o para mal. Mi experiencia personal en este aspecto, me lleva a afirmar que es muy importante que la persona o personas que se hagan responsables de una actividad como esta han de tener muy claro este último punto. Es una reflexión que tarde o temprano va a aparecer, por lo cual hemos de estar preparados para ello. En nuestra emisora de Radio-escuela de Cabretón nos pilló de improviso. Ahora bien, tengo que decir que la reflexión que hicimos en grupo, mis alumnos y alumnas y yo mismo, fue una lección maravillosa de Libertad de Expresión, Censura, Autocensura e Independencia que, con toda seguridad, aún no han olvidado.

\subsection{Objetivos}

Hecha estas reflexiones y todas aquellas que nos pueden surgir a lo largo del proceso, podemos poner en marcha esta fantástica y fabulosa actividad. Los objetivos que podemos conseguir con ella son de incalculable valor pedagógico. Por citar alguno de ellos:

\section{Objetivos generales de etapa:}

- Obtener y seleccionar información utilizando las fuentes en las que habitualmente se encuentra disponible, tratarla de forma autónoma y crítica, con una finalidad previamente establecida y transmitirla a los demás de manera organizada e inteligible.

- Analizar los mecanismos y valores que rigen el funcionamiento de las sociedades, en especial los relativos a los derechos y deberes de los ciudadanos, y adoptar juicios y actitudes personales con respecto a ellos.

\section{Objetivos específicos para el área de lengua:}

- Conocer y analizar los procesos de comunicación de la sociedad actual, reflexionando sobre su dimensión lingüística, sociológica, psicológica, tecnológica, económica, ideológica y política.

- Comprender el funcionamiento y practicar el uso de las tecnologías de la información y la comunicación, manteniendo actitudes reflexivas y críticas sobre las mismas.

- Interpretar el contenido y la intención de los mensajes producidos mediante las tecnologías de la información, descifrando sus elementos formales y estructurales. 
- Utilizar las tecnologías de la información y la comunicación para producir mensajes que integren diferentes lenguajes.

- Valorar su experiencia personal en la producción y emisión de mensajes.

- Orientar las capacidades expresivas y lúdicas de los alumnos/as hacia los tratamientos audiovisuales propios y autónomos que superen la imitación de los modelos establecidos.

- Analizar y valorar los usos indebidos de las tecnologías de la información reconociendo su contenido ideológico.

- Realizar tareas en grupo y participar en discusiones y debates con una actitud constructiva, crítica y tolerante, fundamentando adecuadamente sus opiniones y propuestas y valorando la discrepancia y el diálogo como una vía necesaria para la solución de todo tipo de problemas.

- Apreciar los derechos y libertades humanas como un logro irrenunciable del ser humano y como una condición necesaria para la paz, denunciando actitudes y situaciones discriminatorias e injustas mostrándose solidario con los pueblos, grupos sociales y personas privados de sus derechos o de los recursos económicos necesarios para llevar una existencia digna.

\subsection{Tipos de programas}

Los programas que a lo largo de estos tiempos hemos elaborado han sido innumerables. Todos los alumnos y alumnas de los centros rurales donde he puesto en marcha esta actividad, así como sus correspondientes maestros y maestras, han participado en las diferentes emisiones realizadas. Los padres y madres y pueblos en general, siempre hay honrosas excepciones, han escuchado embelesados a sus niños y niñas.

En el momento en que comenzamos la actividad hubo que plantearse, en relación a los programas, el tipo de alumnado que lo realizaría así como el sentido y finalidad de los mismos. Mi reflexión, personal en este caso aunque haciendo partícipe de ello al alumnado, fue bastante clara desde el primer momento. La radio era una actividad muy enriquecedora para mis alumnos y alumnas, por eso estaba trabajando con ella. Pero no podía olvidar, como ya he citado, la importancia que podría tener para los padres y madres de nuestro alumnado y, por otra parte, para el pueblo en general. Todo esto debería tenerlo en cuenta a la hora de inventar, por decirlo de alguna forma, los programas.

En el caso del alumnado tenía muy claro que los diferentes programas deberían trabajar aspectos relacionados con los contenidos académicos. Era una estrategia para motivarles y, por supuesto, para trabajarlos mejor y con más profundidad. Materias como Conocimiento del Medio, Sociales, Naturales y, sobre todo, Lenguaje se veían beneficiadas con este planteamiento. Pero, además, tenía que aprovechar esta actividad para educar, con mayúsculas, a mis alumnos y alumnas. Tolerancia, respeto a la opinión del otro, libertad de expresión, censura, autocensura, independencia, pensamiento y actitud crítica, toma de decisiones, saber hablar y escuchar y otros muchos aspectos debían ser trabajados también. De ahí la puesta en funcionamiento de todo una serie de programas relacionados con la expresión de la opinión, ya sea propia o la de otros.

En mis años como docente, si algo me ha quedado claro ha sido la importancia de los padres y madres en la educación de los niños y niñas. Están primero en sus manos 
y pasan posteriormente, y unos tiempos muy marcados, a las nuestras. Nosotros estamos para educar. El problema se plantea cuando los padres y madres nos encontramos con dudas, con muchas dudas sobre lo que hacemos. En muchas ocasiones nos planteamos si lo que hacemos o decimos a nuestros hijos e hijas está bien o mal. La sociedad, en general, y los centros educativos, en particular, han buscado diferentes y diversas estrategias para paliar en parte este problema. En el caso que nos ocupa, la radio escolar, me ha servido para trabajar este aspecto. En cada emisión que hemos realizado ha estado presente ese interés por la formación de los padres y madres de nuestro alumnado. Incluso realizamos un programa titulado "ESCUELA DE PADRES" en el que se tocan temas relacionados con la educación de los hijos e hijas, en general, y con el tema escolar, en particular. Resulta, a decir de los padres y madres, un programa muy interesante y que, posteriormente, en formato escrito hemos ido pasando por la revista escolar del centro.

Si nos plateamos la radio escolar como foco cultural del pueblo, según he citado anteriormente, dos son los aspectos a considerar. De un lado, podemos aprovechar para informar sobre aquellos acontecimientos novedosos, importantes e interesantes que hayan acontecido en el propio municipio. Podemos, incluso, hacer uso de una unidad móvil y acudir al lugar de la noticia entrevistando a los autores de la misma. Es una fórmula ideal para que el pueblo tenga información de primera mano desde el Ayuntamiento, Parroquia, Escuela, Asociaciones, etc. Según las actividades de aula, podemos además informar a nuestros vecinos de las noticias más importantes acontecidas en nuestra comunidad autónoma, país y mundo en general. Por otra parte, podemos utilizar la emisora escolar en la formación de nuestras gentes. Por ello, a lo largo de estos años, hemos ido elaborando programas que tenían como finalidad formar personal y culturalmente a los que nos escuchaban. Recuerdo programas de entrevistas en el año 85 sobre el tema de la entrada en la CEE. Recuerdo entrevistas a alcaldes, personas ilustres, jornadas conmemorativas.

Como muestra simplemente podemos citar diferentes programas que a lo largo de estos años hemos elaborado:

- Espacios Informativos: Noticias, Debates, Entrevistas, Reportajes, etc

- Espacios Dramáticos: Radio-teatro, Cuentos y Leyendas.

- Espacios Musicales

- Espacios Culturales: de Medio Ambiente, Temas Sociales, Temas Educativos.

- Espacios de Entretenimiento: Concursos, Juegos.

\subsection{Organización}

La organización, como corresponde a cualquier actividad educativa, ha de estar bien planificada, no es posible dar cabida a la improvisación que es mala consejera en educación. La organización dentro del aula vendrá marcada por una serie de elementos que hemos de analizar y que son diferentes en cada centro. Hemos de tener en cuenta el número de alumnos y alumnas que van a participar, los grupos y niveles a los que pertenecen, los tiempos que podemos utilizar, los espacios de que disponemos, el número de profesores que se van a implicar en el proyecto, los recursos con los que contamos, el valor educativo que vamos a dar a la actividad dentro o fuera del curriculum, las materias que se van a ver enredadas en el tema y todos aquellos aspec- 
tos organizativos que irán surgiendo en el momento que se inicie la preparación y la emisión de los programas.

Mi planteamiento y mi planificación fue bien diferente en la primera época y en la segunda. Bien diferentes eran los centros donde ejercí la docencia. Todos eran rurales, pero el número de alumnos y alumnas y los niveles con los que trabajé en cada uno de los casos los hacía totalmente distintos. Los recursos que conseguimos en el primer caso y con todos los que contábamos en el segundo hacía incomparable la actividad. El grado de interés demostrado por el pueblo en general en uno y otro caso hacía el proyecto más o menos interesante. En definitiva, el sistema organizativo era totalmente distinto en uno y otro caso.

Radio-Escuela de Cabretón ofrecía más posibilidades por el simple de hecho de contar con más alumnado y más niveles. Contábamos con 9 Grupos de Trabajo: 4 de Ciclo Superior de EGB, de Ciclo Medio y 1 de Ciclo Inicial y Preescolar. Existía un Equipo Coordinador formado por tres alumnos de $8^{\circ}$ de EGB que, entre otras funciones, revisaban toda la programación, asesoraban a los compañeros, corregían los guiones y grababan los programas en diferido. Con el fin de que los propios alumnos pudieran escuchar a sus compañeros y, sobre todo, para evitar la monotonía y el cansancio teníamos dos Grupos de Grabación que se alternaban semanalmente en la elaboración y emisión de los programas.

En el caso de Radio-Villoslada, toda la organización pasaba por tres Grupos de Trabajo: uno de la ESO, otro de Educación Primaria y el último a Educación Infantil. Además los dos últimos grupos solamente participaban en ciertos programas. Todo el peso de la actividad descansaba en el grupo de la ESO que, a su vez, se dividía en dos subgrupos de 3 y 2 alumnos respectivamente. Ante esta situación, si en el caso de Cabretón emitimos semanalmente una hora sin faltar nunca a la cita, en el Centro de Villoslada llegamos a realizar 8 emisiones, aunque con una duración aproximada de tres horas cada una. En el caso de radio Ortigosa, la existencia de un número de alumnos/as suficientes me ha permitido formar diferentes grupos de trabajo al estilo de La Radio de Cabretón. El uso del ordenador, a la hora de planificar y trabajar, nos ayudó mucho en los últimos casos de Villoslada y Ortigosa. Contamos con una serie de fichas que nos facilitan enormemente nuestro trabajo.

La Hoja de Emisión informa de los programas que hemos de elaborar y sus características técnicas: tiempos, hora de emisión, responsables y tipo de programa.

La Hoja de Trabajo permite conocer a cada grupo los programas elaborados y su tipología. Es una forma de controlar que todos los grupos trabajen más o menos lo mismo y que elaboren de todo tipo de programas.

Contamos con una Hoja de Evaluación de Programas que nos permite recoger el resultado del análisis realizado por todos los alumnos/as sobre los diferentes programas.

Por último, disponemos de una Hoja de Evaluación de Alumnos/as donde voy realizando las anotaciones correspondientes a las diferentes actividades realizadas por todo el alumnado.

A decir verdad, el romanticismo y mis recuerdos sobre la radio escolar me trasladan a Cabretón pero la calidad técnica me habla de Villoslada. En ambos casos disfruté mucho como docente. En los dos colegios, mis alumnos y alumnas me consta que disfrutaron y, lo que es más importante, en ambos aprendieron muchas cosas. Es una señal, una marca que llevaremos siempre dentro, en nuestra mente. 


\subsection{Evaluación}

Uno de los momentos fundamentales en todo acto educativo es, en mi opinión, la evaluación. Por ello, después de cada una de las emisiones realizadas, Ilevábamos a cabo una sesión de reflexión sobre todo lo realizado. En este aspecto, tanto en mi primera experiencia en Cabretón como en la segunda de Villoslada y Ortigosa, el sistema de evaluación es similar.

- Los aspectos a analizar en cada una de las emisiones eran muy claros y sencillos:

- Nivel de calidad del control técnico de la emisora

- Locución por parte de todos los participantes en la emisión

- Los tiempos reales dedicados a cada uno de los programas en comparación con lo establecido.

- La calidad de los programas en cuanto a sus contenidos.

- La participación, motivación y actitud de todos los participantes en la actividad.

- El proceso de evaluación es bastante sencillo:

- Audición de la emisión grabada al respecto.

- Individualmente, toma de notas según los criterios señalados anteriormente y conocidos por todos.

- En grupos, puesta en común de los datos tomados y conclusiones.

Todas las conclusiones quedan reflejadas en la Hoja de Evaluación correspondiente que es colocada en el tablón de anuncios para conocimiento de todos nosotros.

\subsection{Estudiar la radio}

Para poder iniciarse en la elaboración de programas de radio, es del todo imprescindible poseer y manejar una serie de herramientas educativas para lo cual es necesario que en el aula introduzcamos, en aquellos momentos que establezcamos, unos contenidos básicos. Es el tiempo dedicado al estudio de la Radio como medio de comunicación. Es, por decirlo de alguna manera, el momento de la teoría. No se trata de iniciar la eterna discusión de teoría y práctica. En este caso, como en todos, ambos se necesitan, quizás discrepamos los docentes del momento de su aplicación.

En Radio-Escuela de Cabretón, a decir verdad, no hubo teoría en sus inicios. Nuestro tiempo se dedicaba por completo a la práctica. Pronto observé la necesidad de trabajar una serie de aspectos que mejorarían notablemente las emisiones. Radio Villoslada y Radio Ortigosa contó, desde sus inicios, con toda la experiencia anterior lo cual supuso que teoría y práctica fueran parejas como no podía ser de otra manera. Por ello elaboré una serie de Unidades Didácticas que a lo largo del primer trimestre, sobre todo, y del segundo vamos trabajando en el aula. Nos sirven para conocer aspectos interesantes y necesarios a fin de poder realizar los diferentes programas. Pero también nos sirven para confirmar que muchas de las cosas que estamos haciendo, las hacemos bien.

Los títulos de las Unidades trabajadas que trabajamos son las siguientes:

* Unidad no 1: "La radio: funcionamiento y organización"

* Unidad no 2: "El lenguaje radiofónico, la locución"

* Unidad no 3: "Conceptos básicos de radio. Música y efectos especiales" 
* Unidad no 4: "El guión"

* Unidad no 5: "Las fuentes de información"

* Unidad no 6: "Tipos de programas"

* Unidad no 7: "La entrevista"

Para el estudio de estas unidades preparé, en su momento, una serie de materiales entresacados de diferentes libros que he ido buscando por la biblioteca del centro, del CPR y otros materiales que poseo recopilados durante estos años.

\section{Valoración del uso de la radio escolar}

A lo largo de estos años he ido comprobando las ventajas pedagógicas que el uso de la radio escolar ha supuesto a todos aquellos alumnos y alumnos que durante este tiempo se han beneficiado de esta actividad. Sería interesante preguntarles a ellos, hoy en día y años después, acerca de esta experiencia que sufrieron en sus carnes. Yo no lo he hecho de forma directa. Simplemente, en los encuentros casuales con ellos, siempre ha habido un recuerdo para aquella radio que inventamos entre todos.

Sin embargo, creo que es de recibo dejar plasmado en este trabajo todo aquello que fuimos logrando entre todos. Se hace necesario, para todas aquellas personas que estén interesadas en el tema, hacer una valoración y entresacar aquellos aspectos que, sin duda alguna, fueron mejorando con la práctica diaria del uso de la radio en el aula.

De todas las formas, estoy convencido de que esta actividad altamente motivadora y eminentemente educativa de poco puede servir si no se encuentra inmersa en un sistema de trabajo y en una forma de entender la educación determinados. De nada servirá hacer radio en un sistema donde prime el autoritarismo y el mal ambiente, donde no se permita la participación y la exposición de las propias ideas, donde se valora más el aislamiento del alumnado, donde se evalúe por encima de todo los conceptos y los conocimientos.

\subsection{Motivación}

Es, a mi modo de ver, el elemento que más favorecido sale en esta valoración. El aula sufre un cambio radical. La escuela en su conjunto se siente mucho más dinámica y activa. Todas las aulas del centro que participan en el proyecto mejoran su nivel motivacional. Toda la comunidad educativa, especialmente las familias, sienten la escuela mucho más cerca. La radio supone un medio de contacto con el mundo escolar, con el aula, con el profesorado. Tanto el alumnado como el profesorado y, si se me permite, toda la comunidad educativa se siente especialmente motivada con esta actividad. Todo ha redundado evidentemente en una mayor implicación de toda la comunidad en el hecho educativo. Además, puedo afirmar con toda rotundidad que todo el alumnado ha mejorado notablemente su visión de la escuela y de las actividades escolares, especialmente la que nos ocupa. Todo ello ha supuesto una mejora paulatina en el ambiente de aula y de centro.

\subsection{Cambios metodológicos}

Introducir el uso de la radio en la escuela y en sus aulas ha supuesto para mí una serie de cambios a nivel metodológico que me ha llevado a replantearme seriamente mis formas y maneras educativas anteriores. Me ha llevado a replantearme aspectos 
tan importantes como la organización del aula, la utilidad de los contenidos, el uso de los agrupamientos de alumnos y alumnas, las técnicas de trabajo a emplear en el día a día, la inclusión de los valores y las normas en la programación de aula, los espacios educativos y sus tiempos, las actividades y los deberes $y$, de una forma muy especial, la participación del alumnado en el aula y en la escuela. A nivel personal, esta actividad ha sido un buen estímulo para reflexionar sobre mi actuación diaria en el aula. Sin duda, no es necesario hacer radio para reflexionar. Ahora bien, hacer radio te hace meditar y buscar nuevas estrategias, nuevas fórmulas, más imaginativas y más activas, que supondrán un cambio en nuestras maneras y una mayor motivación en nuestro alumnado. Me ha ayudado a perder el miedo a lo nuevo, a lo diferente. Me ha permitido innovar, investigar $y$, en definitiva, progresar.

\subsection{Mejora en los procesos de aprendizaje}

A lo largo de estos años, puedo decir que he observado cómo mis alumnos/as han ido paulatinamente mejorando muchos aspectos que, estoy seguro, habrían mejorado también con otras muchas actividades, incluso las más retrógradas. Sin embargo, me atrevo a afirmar que lo han hecho con mucho entusiasmo, con inmensa alegría, con muchas ganas $y$, sobre todo, con mucho trabajo y esfuerzo porque si algo supone esta actividad es precisamente eso: trabajo y esfuerzo.

Mejorar el nivel de lectura comprensiva y expresiva es y ha sido uno de los grandes objetivos que siempre me he planteado con esta actividad. Es, sin lugar a dudas, la gran beneficiada. Los alumnos y alumnas han comenzado a comprender la importancia de leer bien cuando se les ha puesta en situación real de comunicación. Han entendido entonces que es necesario mucho esfuerzo y mucho sacrificio para leer bien, pero que todo ello tiene una gran recompensa personal. Con esta actividad se empiezan a dar cuentas de aspectos de la lectura tan inteligibles para ellos, pero tan importantes, como: la vocalización, la dicción, la entonación, el ritmo, la velocidad, la comprensión, la vacilación, las rectificaciones, las supresiones, y otras muchas dificultades que analizamos en cada emisión. Ser conscientes de todo ello es el primer paso para su corrección.

Ése es el principio. A lo largo del tiempo, vamos trabajando también de una manera muy especial la Expresión Escrita, aspecto que, a mi modo de ver, es fundamental en los niveles en que nos movemos. Las narraciones para los programas especializados, la elaboración de diferentes textos, la realización de reportajes y noticias propician unos procesos de trabajo en los que la lengua es el elemento clave. El tiempo me ha hecho observar cómo todo el alumnado va elaborando textos de una mejor calidad. Todos los alumnos/as salen fortalecidos ya que todos ellos van trabajando en sus respectivos grupos de acuerdo a sus capacidades y posibilidades. La estructuración de estos textos, la reflexión sobre temas diversos y su redacción permiten al alumno organizar de forma adecuada su mente y empezar a ser conscientes de lo importante que es tener su propio criterio, su personalidad, ser críticos, etc...

El uso de las técnicas de trabajo intelectual es permanente en el trabajo con la radio escolar. La búsqueda de materiales adecuados en la biblioteca o en internet es una constante. Esto favorece la introducción de las nuevas tecnologías y el hacer un uso adecuado de ellas. Por otra parte, el alumnado aprende a usar la biblioteca correctamente. Seleccionar textos es otra de las fases interesantes a la que seguirá la fase de elaboración de los guiones. Todo lo anterior ha permitido que mis alumnos/as 
hayan adquirido una mayor autonomía en el trabajo y que hayan desarrollado toda una serie de estrategias que les permiten adaptarse a nuevas circunstancias por difíciles que puedan resultar en principio. Han adquirido toda una serie de mecanismos y de estrategias que les permite diseñar y elaborar diferentes tipos de trabajos en los que es necesario poner en marcha aspectos tan importantes como la iniciativa, la autonomía, la creatividad, la originalidad, la organización, la selección, etc...

\subsection{Educación en valores}

Con la llegada de la LOGSE, se hizo más patente que nunca la necesidad de educar a nuestro alumnado en el mundo de los valores, actitudes y normas de una manera clara y sin ambigüedades. Todo eso que resulta muy bonito e interesante sobre el papel, era necesario ponerlo en marcha en los centros y en las aulas. La actividad de la radio escolar me ha permitido trabajar muchos de estos contenidos de una manera explícita. Hemos tenido que reflexionar en muchas ocasiones acerca de conceptos tan interesantes como: libertad de expresión, censura y autocensura, ideología e independencia, manipulación e intoxicación, respeto y tolerancia. Todo ello ha sido trabajado en nuestras aulas y el alumnado es consciente de lo que todo ello significa. En muchos momentos hemos tenido que tomar decisiones propias de los grandes medios de comunicación.

Me gustaría hacer un especial hincapié en un aspecto que yo considero muy importante en la educación de nuestros niños y jóvenes, que no es otro que el trabajo en equipo. Introducir la radio en el aula, como ya he citado anteriormente, ha supuesto una serie de cambios metodológicos importantes, uno de estos cambios tiene que ver con la formación de grupos de trabajo. La colaboración, la cooperación, el respeto a los demás y a sus ideas, la tolerancia son valores que se aprenden con la práctica diaria de los mismos. En nuestro caso y con el uso de la radio, esto es constante. La mejoría en las relaciones entre el alumnado es evidente y palpable. Esto ha supuesto para el aula y para el centro un ambiente educativo y de estudio inmejorable. Hay que destacar en este aspecto que los padres y las madres son, además, conscientes de ello y nos lo hacen saber. Nuestra aula es un aula dinámica y activa, y está organizada de tal forma que en cualquier momento podemos colocarnos en diferentes grupos de trabajo sin necesidad de producir grandes molestias y en pocos segundos.

\subsection{Participación}

En mi opinión, es fundamental, para el buen funcionamiento del aula y del centro, que todas las personas que formamos la comunidad educativa nos impliquemos en las diferentes actividades que se programen. Entender este planteamiento es especialmente importante ya que, de lo contrario, la filosofía acerca del uso de la radio en la escuela, en mi opinión, se desvirtuaría totalmente. Con esta actividad mejora notablemente la participación de los alumnos/as en las actividades de radio, en particular, y en el aula, en general. Sin su efectiva colaboración y su implicación directa, pocas cosas podemos hacer. Esta participación se lleva a cabo en los diferentes momentos de la actividad. En el momento del diseño del sumario de la emisión, ellos aportan ideas sobre diferentes programas que podemos realizar. Muchos de los programas que elaboramos son ideas de los propios alumnos y alumnas. Nada se les da hecho, todo desde ese momento es cosa de ellos, con el consiguiente asesoramiento y apoyo. Participan muy especialmente en la evaluación de las emisiones. Son los primeros que 
analizan y valoran aquello que han hecho a lo largo de la emisión. Comprueban desde los primeros momentos las dificultades que tienen, los problemas que han surgido, las causas de los mismos y la forma y manera de rectificar buscando las soluciones adecuadas. Esta sesión de evaluación que realizamos después de cada emisión, es muy interesante para todos, especialmente para los alumnos y alumnas. De todo ello dejamos constancia escrita a fin de que no se nos quede en el tintero de cara a posteriores emisiones.

\subsection{Relaciones interpersonales y ambiente}

No voy a descubrir nada especial si afirmo que el ambiente de aula y la dinámica que en ella se establezca, son elementos básicos para que el acto educativo se desarrolle de la forma más adecuada.

Como resumen a todo lo positivo que esta actividad aporta al aula y al centro, puedo decir que el ambiente y las relaciones entre todos los miembros de la comunidad educativa salen favorecidas, en líneas generales.

Las relaciones entre los alumnos/as mejoran notablemente así como entre todos los individuos que participamos activamente en la actividad. La dinámica del aula es diferente. Muchas de las actividades que realizamos en las diferentes materias pueden formar parte de las emisiones de radio lo cual les confiere un interés especial por parte del alumnado. El sector de padres/madres se sienten identificados con la actividad. A ellos va dirigida, en ellos pensamos al realizar muchos de los programas, se encuentran puntualmente informados de lo que realizamos y colaboran en determinados momentos con la escuela. Esto hace que las relaciones entre escuela y familias sean más estrechas y favorezca la comunicación, aspecto que en mi opinión es muy beneficiosa para nuestro alumnado que son sus hijos e hijas. Este proyecto mejora también las relaciones entre el profesorado implicado en la actividad. Sentimos que formamos parte de un proyecto común en el que cada uno realiza su aportación personal. Es algo diferente a lo habitual, a las reuniones de equipos o departamento. Todos nos vamos enriqueciendo mutuamente.

\section{Referencias bibliográficas}

RíO APARICIO, P. (1990): La radio en el diseño curricular. (Colección Nueva Escuela). Madrid, Ed. Bruño.

GÓMEZ PALACIOS, J. J. (1987): La radio joven. Madrid, Ed. CCS.

GONZÁLEZ, F. (1989): En el dial de mi pupitre. (Colección Medios de Comunicación en la enseñanza). Barcelona, Ed. Gustavo Gili.

MARTíNEZ REYES A. y otros (1993): La radio escolar. Cádiz, Colección EDU.CA. 